\title{
IMPACT OF SORTING MACHINE ON LIFE CYCLE OF PASSIVE UHF RFID TAGS PLACED ON LETTER MAIL
}

This article deals with research of passive RFID technology in conjunction with letter-size mail, which is placed into sorting machine. RFID technology is a part of automatic identification and data capture. In this article we would like to describe an identification of letter mail based on passive technology. We would like to specify, how the sorting machine impacts a readability of RFID tags placed on letter mail. We have established a number of variants of placing RFID tags into envelopes. After passing these tags, we evaluated their readability. Then we compared the results before and after passing the letters through the sorting line. All results are verified by measurement at the Slovak post main processing center located in Zilina. The results of our research bring the new point of view and indicate the ways of using of UHF RFID technology in postal and logistics applications. At the end of this article we evaluate the impact of the selected sorting line on a life cycle of RFID tags.

Keywords: RFID technology, sorting machine, passive tag, letter mail, identification.

\section{Introduction}

This article deals with research of life cycle of passive RFID tags passed through the sorting machine. We performed research of readability of RFID tags in different conditions of selected parameters. Areas of application of RFID technology are also postal and logistic processes. In this context there are several questions of feasibility of the use of identification of letters, parcels etc. Today, postal operations have implemented RFID in various closed-loop systems to measure, monitor, and improve operations. For example, RFID is used to monitor international mail service between major hubs. By randomly "seeding" tagged letters into trays, elapsed delivery time can be measured. This allows service issues to be identified and addressed in a reliable and cost-effective manner. By allowing information to be captured automatically, RFID makes sure it is done, even under stressful conditions.

\section{Objective and methodology}

Objects of the research were the transport items (letter mail) and passive RFID identifiers placed into thesetransport units. RFID tags were read by passive RFID readers in several positions and conditions. In order to achieve the relevant results of the research (Fig. 1), more than 100 measurements were performed. Measurements were realized by various types of testing.

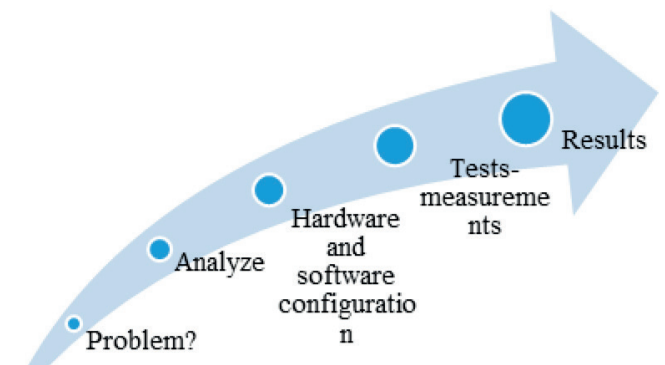

Fig. 1 Steps to achieve good results

\section{Theoretical background}

\subsection{RFID system}

The RFID system architecture consists of a reader and a tag (also known as a label or chip). The reader queries the tag, obtains information, and then takes action based on that information. That action may display a number on a hand held device, or it may pass information on to a POS system, an inventory database

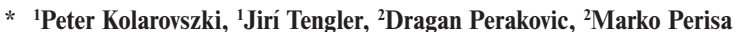

${ }^{1}$ Department of Communications, Faculty of Operation and Economics of Transport and Communications, University of Zilina, Slovakia

${ }^{2}$ University of Zagreb, Faculty of Transport and Traffic Sciences, Croatia

E-mail: kolarovszki@fpedas.uniza.sk
} 
or relay it to a backend payment system thousands of miles away. Let's take a look at some of the basic components we have used in our research.

RFID tag is a small device that can be attached to an item, case, container, or pallet, so it can be identified and tracked. It is also called a transponder. The tag is composed of microchip and antenna. These elements are attached to a material called a substrate in order to create an inlay [1].

Tags are categorized into three types based on the power source for communication and other functionality.

Active.

Passive.

Semi-passive.

RFID interrogators (often called readers) which are devices that wirelessly communicate with tags to identify the item connected to each tag and possibly associate the tagged item with related data. Both the tag and interrogator are two-way radios. Each has an antenna and is capable of modulating and demodulating radio signals [2].

Middleware is software that controls the reader and the data coming from the tags and moves them to other database systems. It carries out basic functions, such as filtering, integration and control of the reader. RFID systems work, if the reader antenna transmits radio signals. These signals are captured by tag, which corresponds to the corresponding radio signal. This is a very special software device enabling mutual communication between two and more applications. This device is marked also as a mediator between various application components [3].

\subsection{Characteristic of sorting machine}

Compact reader sorter (CRS) provides cancelling on mail pieces which are aligned on their bottom edges. It also provides address reading and videocoding, barcode reading and printing techniques (Fig. 2). The main focus is to sort letter mail items up to 24 stackers in inward and outward sorting options [4].

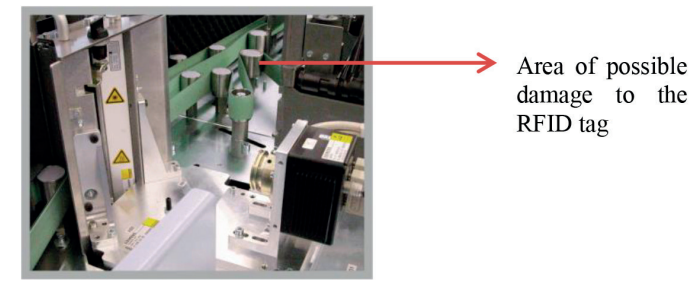

Fig. 2 Possible damage zone [5]

\subsubsection{Address reading}

In order to recognize the addresses of the mail pieces, which are running through the machine, a letter scanner scans an image of each mail piece. To achieve this, the mail piece is illuminated and the reflected light is photographed with a camera. The scanner electronics converts the gray image into a binary image. The scanner passes the image on to the reader electronics (IP-PC) [5 and 6].

Furthermore, the scanner passes on image-accompanying data e.g. in which areas of the image the address should be searched for. Mail pieces, which are correctly aligned and whose addresses can be read and recognized, are sent to a designated stacker. If the address of a correctly aligned mail piece can not be read, the machine control computer directs it to a special sorting stacker. If a video coding unit is connected to the system the address is coded manually and thus allows later refeeding of the mail piece. For this purpose the mail piece must be additionally tagged with an ID-tag in a different unit of the system [7 and 8].

\subsubsection{Barcode reading and printing}

The mail pieces pass by a faceplate with an integrated scanning zone. Two reflection light barriers in the faceplate determine the beginning of the mail piece and check the correct height orientation of the mail piece. The bars of the barcode are lit fluorescently through LED lighting. This optical signal is recorded with an arrangement of lenses, changed into an electric signal and forwarded to the evaluation electronics. The evaluation electronics transfers the results to the control computer, via a serial RS232 interface. The control computer directs each mail piece, in accordance with the sorting plan, to a stacker.

A destination barcode or an ID barcode printed on the mail piece identifies the mail piece and contains information like e.g. serial mail piece number. This makes it possible to determine the address of the mail pieces "off-line". If the machine can not read the addresses itself, it can forward the image of the mail piece to an off-line Video Coding System [9].

\section{Description of measurements}

\subsection{Scenario of measurement}

Scenario of measurement was realized by two levels. First level deals with testing of RFID tags on logistic units before and second level after the selected physical effects. In this way we gained the status of RSSI and read count before any damage to the RFID tag and after the possible damage. The measurements were realized under the same conditions, before and after damaging the RFID tags, in a single cycle, i.e. linear line (100 of transitions). Speed of transition of RFID tags on the linear line and recording period information was selected on the basis of secondary research, which served as a starting point for selecting these values. In the secondary measurements we combined rate 
of passage through the RFID gate by the linear line ranging from 0.100 meters per second up to 2 meters per second. We set the period (latency possible load) from $0 \mathrm{~ms}$ and $2500 \mathrm{~ms}$ by using the antenna in a horizontal, vertical and then horizontal and vertical position. From the total number of combinations in terms of the RSSI, the read count and used antennas, the combination of period 0 with speed 0.7 meters per second, and one antenna in a vertical position was selected. This selected combination was used in the primary measurement. Accurate placement of RFID antennas within linear line realized by secondary and primary measurement is shown in Figs. 3 and 4.

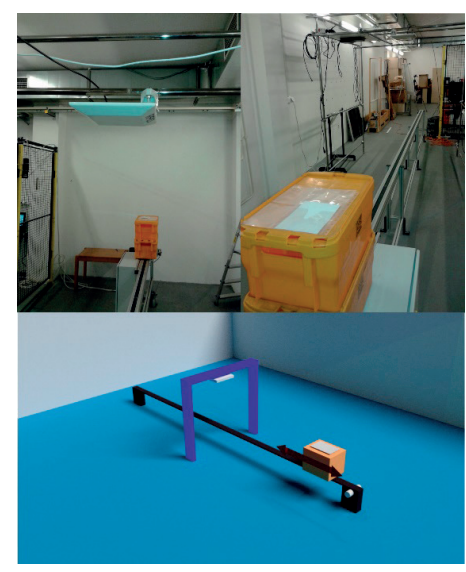

Fig. 3 Linear line / conveyor

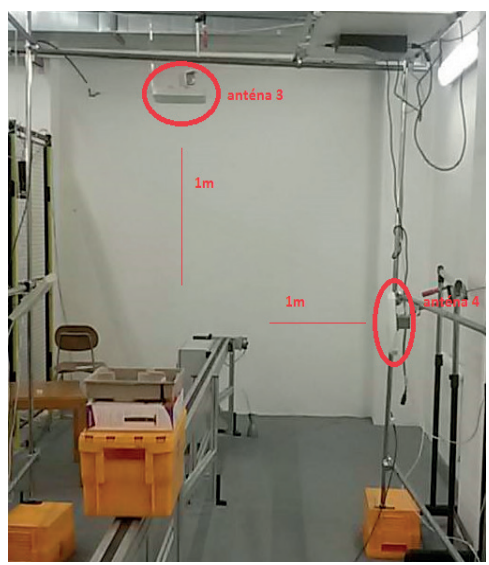

Fig. 4 Placement of antennas

\subsection{Physical effects}

On the letter mail with RFID tags following physical effects were tested:

1. Impact of frost (by the transportation in the winter time)

2. Impact of the magnetic and electromagnetic fields (during a contact with mechanized equipment possibly with other shipments)

3. The effects of water (after loading and unloading time)

4. Impact of the moisture (in case of rain and drizzle)

5. Exposure to high temperatures 40 and 60 degrees (by the transportation in the summer time)

6. Pressure (contact and friction with other letter-size mail)

7. Damage of the RFID tag (tearing of the label apart due to poor handling or contact with other letter mail)

8. Impact of sorting line

\subsection{Damaging of RFID tags}

The physical effects were realized in different environments. The first seven types of physical effects were realized under laboratory conditions. The last physical effect was realized in the postal sorting center of letter mail.

\section{Measurement results}

\subsection{Result of damaging of RFID tags under laboratory conditions}

As mentioned above we focused on two specific parameters and those were RSSI value i.e. average value within one cycle and the read count of RFID tags within one cycle. Before starting with the trial results, it is necessary to mention that we worked with the starting and end values of RFID labels as a single unit. The reason was that every RFID tag is a bit different. That is the reason that starting values for each label are different, sometimes even dramatically. In Fig. 5 the read count of RFID tags before and after impact of physical influences can be seen. Based on the results of the measured values we can confirm these conclusions. The biggest differences we found out in the impact of frost over the period of 72 hours (RFID tag was frozen for 72 hours). Also results with the frost-free period of 24 and 48 hours were not negligible. Differences were on average $72 \%, 41 \%, 36 \%$. We observed also the big differences after impact of electromagnetic fields and exposure to high temperatures (40 to 60 degrees) within one hour of measurements. Differences were on average $56 \%, 49 \%, 46 \%$. We recorded lower differences in the impact of water and steam, or moisture, where the difference between two impacts was $36 \%$. Surprisingly, the lowest differences were seen in impact of classic magnet and neodymium magnet even under 
pressure. The difference was $1 \%, 11 \%$, and at a pressure $2 \%$, what is negligible.

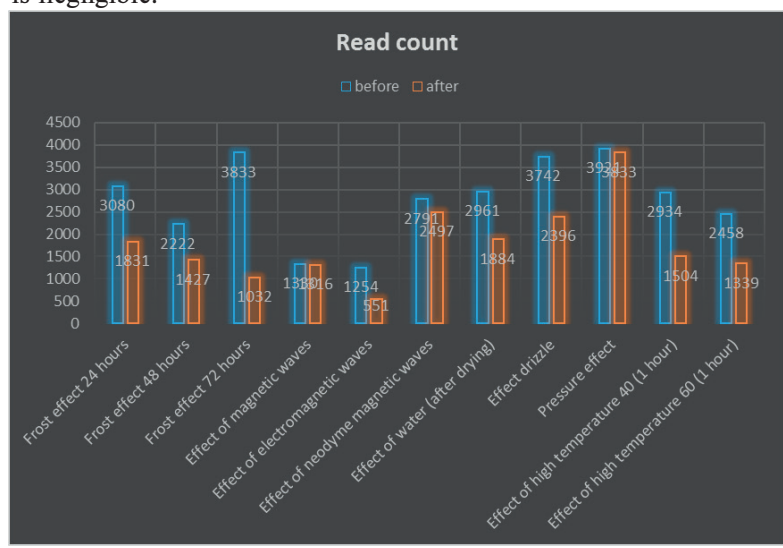

Fig. 5 Read count value before/after

In the measurement and result evaluation, we evaluated a second parameter which was RSSI (Fig. 6). In terms of the measured range we can ' $t$ make any justified conclusions, because differences in terms of the measured range are quite negligible. So we can say that RFID tags mostly maintain its radiation properties.

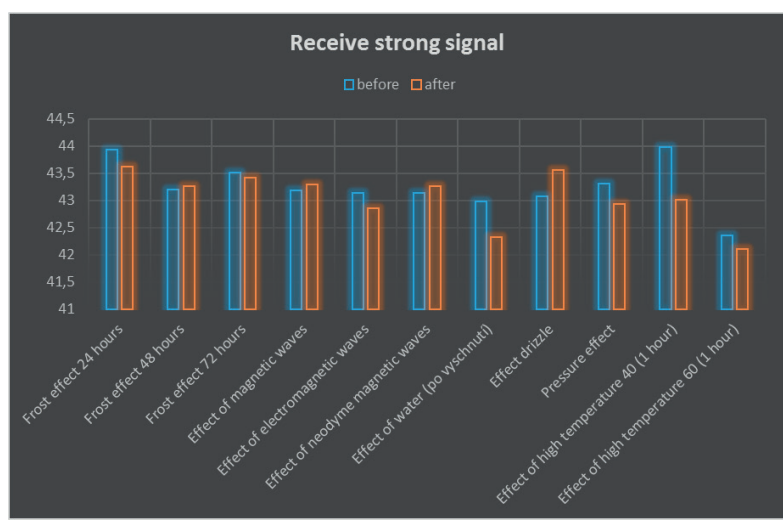

Fig. 6 RSSI value before/after

\subsection{Result of damaging of RFID tags in CRS}

As in the previous case, again we focused on RSSI value and read count (Fig. 7). The most differences were recorded in letter mail that passed through the sorting line two times with one and three sheets of A4. In this case the value of count read decreased by $53 \%$ and $70 \%$. In other cases, we observed a decrease in values between $8 \%$ and $24 \%$.

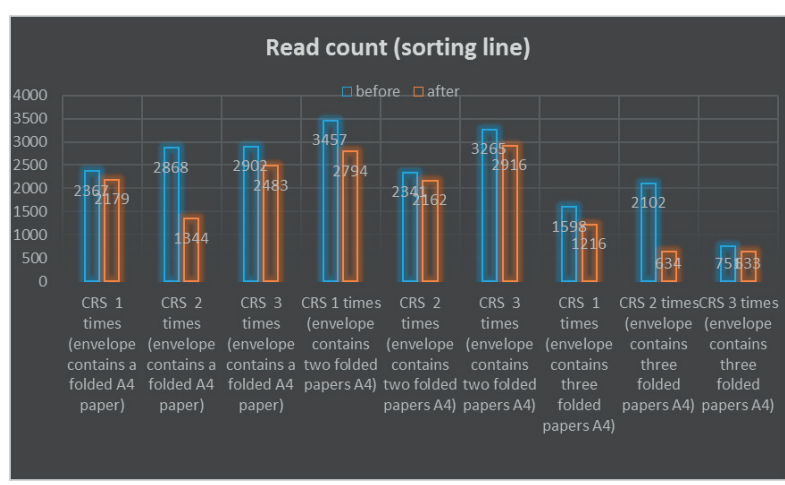

Fig. 7 Count tags read (sorting line)

In our measurement we again focused on RSSI values, but we could not unequivocally confirm the impact of the physical effect through the sorting line. Differences in values (Fig. 8) before and after the sorting line are minimal.

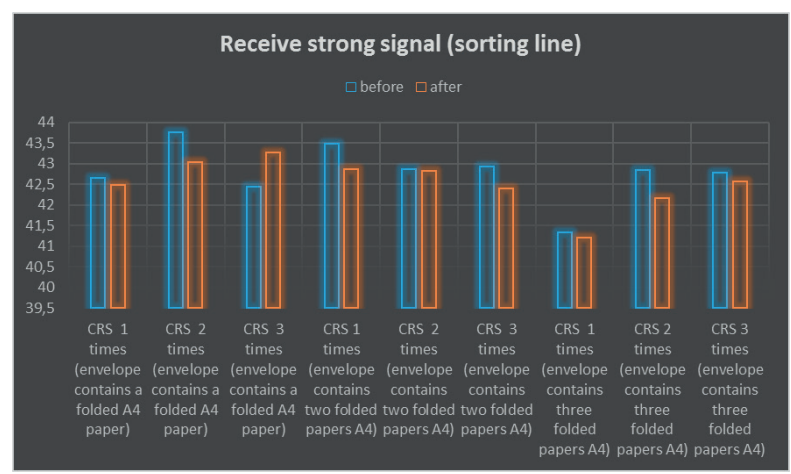

Fig. 8 RSSI value before/after (sorting line)

\section{Conclusion}

The aim of this article and also related measurements was to demonstrate the effects of selected forms of physical effects which the letter item fitted with RFID tag can meet with during the transportation. As part of the recorded values we obtained read count number and RSSI value. We made two measuring parts, first part was before and second after impact of physical effects. The difference between these two measuring parts subsequently provided the requested data. For the first scale of data (read count of RFID tags), the differences were so big that we could clearly, for most of their physical effects, confirm the negative effect of RFID tag. Despite the large decrease in the read count of each tag, these tags still remained powerful and capable of working. Within the measurements we compared these with the value of RSSI. With this type of measurements, the differences were so small that we could not conclude any clear conclusions, since the majority of RFID tags quality signal didn't change significantly from the antenna of RFID tag. Therefore, we were able to prove 
uniquely only the impact of physical effects in terms of read count that was in frost condition (effect after 72 hours) $73 \%$. The impact of the sorting line was strongly observed in only two of the nine options. Similar results were seen in RSSI value. Based on the results of the impact of the sorting line on RFID tag, we can only assume that there is a physical effect, but this situation probably occurs randomly. Physical effects were acting only once on individual tags. In this case we can say, that read count and RSSI values were stabled even more by the repeated impact of physical effects.

\section{Acknowledgement}

This article was created to support project named as:
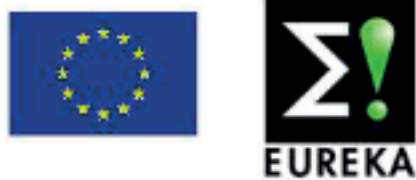

E! 7592 AUTOEPCIS - RFID Technology in Logistic Networks of Automotive Industry

Centre of Excellence for Systems and Services of Intelligent Transport II ITMS 26220120050 supported by the Research \& Development Operational Programme funded by the ERDF.

\section{References}

[1] HUNT, V., PUGLIA, A. AND PUGLIA M.: RFID: A Guide to Radio Frequency Identification. New Jersey : John Wiley \& Sons Inc., 2007, $201 \mathrm{p}$.

[2] VOJTECH, L., NERUDA, M.: Application of Shielding Textiles for Increasing Safety Airborne Systems - Limitation of GSM Interference, $9^{\text {th }}$ Intern. Conference on Networks (ICN 2010), Los Alamitos : IEEE Computer Society, 2010, pp.157-161, ISBN 978-0-7695-3979-9.

[3] MADLENAK, R., MADLENAKOVA, L., STEFUNKO, J.: The Variant Approach to the Optimization of the Postal Transportation Network, The Conditions of the Slovak Republic, Transport and Telecommunication J., vol. 16, No. 3, pp. 237-245, ISSN (Online) 1407-6179, June 2015.

[4] VOJTECH, L.: Feasibility of RFID Signal Denoising Using Neural Network. Advances in Electrical and Electronic Engineering, vol. 8, No. 5, 2010, pp. 111-113. ISSN 1804-3119. DOI: 10.15598/aeee.v11i5.889.

[5] Technical Description, CRS_018enT_a (C) Siemens AG 2008 / Date of issue: 2008-01-21

[6] SUlgan M., SOSEDOVA, J.: Procurement of Materials and Components for Manufacturing Activity, Communications - Scientific Letters of the University of Zilina, vol. 16, No. 2, 2014, pp. 41-45, ISSN 1335-4205.

[7] BENES, F., KUBAC, L., STASA, P., KEBO, V.: RFID and Augmented Reality, Proc. of the 14th Intern. Carpathian Control Conference (ICCC), Rytro, May 2013, Vienna : IEEE, 2013, pp. 186-191, ISBN: 978-1-4673-4489-0.

[8] KUBASAKOVA, I., KAMPF, R., STOPKA, O.: Logistics Information and Communication Technology, Communications - Scientific Letters of the University of Zilina, vol. 16, No. 2, 2014, pp. 9-13, ISSN 1335-4205.

[9] DROZDZIEL P., KRZYWONOS L., MADLENAK R., RYBICKA I.: Selected Aspects of Analyses of Failure Rates of Active Safety Systems in Buses, Communications - Scientific Letters of the University of Zilina, vol. 16, No. 3, 2014, pp. 114-119. 